\title{
Micro-Positioning of Linear-Piezoelectric Motors Based on a Learning Nonlinear PID Controller
}

\author{
K. K. Tan, Member, IEEE, Tong Heng Lee, Member, IEEE, and Huixing X. Zhou
}

\begin{abstract}
In this paper, a learning nonlinear proportional integral derivative (PID) controller is developed for vaguely modeled nonlinear systems under the influence of significant disturbance and noise. The control scheme is generic in nature, but it is applied specifically to the micropositioning of linear-piezoelectric motors in this paper. The design of the control scheme does not require a full mathematical model of the nonlinear system. Simulation and experimental results are provided to highlight the good motion control performance achieved from the control scheme.
\end{abstract}

Index Terms-Learning control, micro-positioning, nonlinear PID control, piezoelectric actuators.

\section{INTRODUCTION}

$\mathbf{P}$ IEZOELECTRIC actuators are innovative manipulators which have shown a high potential in applications requiring manipulation within the submicrometer or even nanometer range. Initial applications have been mainly to provide accurate short travel motion (e.g., microscope focusing device [1]), but efficient cooperated use of multiple piezoelectric elements have expanded the application domain to include longer travel applications as in microassembly (e.g., MEMS), precision metrology, and process automation.

There are two main classes of linear-piezoelectric motors (LPMs), classified according to their structures and driving principles. The first class works on a direct-drive principle. Deformations of a piezoelectric element are directly used to drive the load for precise positioning [2]-[4]. The main characteristics of the direct-drive piezoelectric motor are: extremely high resolution and nanometer grade positioning precision, short stroke which is generally under $500 \mu \mathrm{m}$, and a high bandwidth. The second class of LPM operates instead on an indirect-drive principle. Several direct-drive piezoelectric actuators work in synchronization to produce a resultant linear motion of the load. In [5], [6], inchworm types of indirect-drive piezoelectric motors are presented which comprise at least three direct-driven piezoelectric actuators. Two actuators act as clamps, determining the direction of travel. The third actuator expands and contracts along the motor shaft when input voltage is applied, thus executing the motion. The generated force and maximum moving speed are very low with this configuration, although there is no physical limitation to travel length and high resolution of five nanometers has already been achieved

Manuscript received October 4, 1999; revised May 7, 2001. Recommended by Technical Editor T. Fukuda.

The authors are with the Department of Electrical and Computer Engineering, National University of Singapore, Singapore 117576 (e-mail: eletankk@nus.edu.sg; eleleeth@nus.edu.sg; elezhx@ nus.edu.sg).

Publisher Item Identifier S 1083-4435(01)10727-1.
[4]. Another type of indirect-drive piezoelectric motor is the ultrasonic motor or the acoustic motor which uses piezoelectric components to generate ultrasonic waves and produce a linear motion [7]-[10]. The characteristics of ultrasonic piezomotors are: high resolution, unlimited travel, wide dynamic range of velocity, hold stability at power off, and a small compact structure. In this paper, we are mainly concerned with this type of indirect-drive LPM.

While the piezoelectric actuator has high potential for applications in ultraprecision motion control systems, the highly nonlinear features associated with the dynamics of these elements are challenges to how efficiently these potentials can be realized. For the direct-drive type, hysteresis is the main part of the nonlinear characteristics [11]. For the indirect-drive type, friction has been identified as the main problem to be addressed [7], [12]. From the viewpoint of the control system, friction poses an interesting and challenging dilemma to the control problem. On one hand, it provides the primary transfer mechanism to bring about the motion, and on the other hand, it opposes the realization of precision motion control. The frictional characteristics associated with servomechanisms are highly nonlinear in nature, and a good friction model is especially important for applications involving high precision motion control of servomechanisms, where the frictional force needs to be adequately compensated in order to improve the transient performance and to reduce steady-state tracking errors. Model-based approaches are usually proposed to for friction compensation. Even in adaptive control of servomechanisms, an initial friction model is also crucial to ensure smooth control signals and rapid parameter convergence [13]. However, friction modeling has always been a difficult and challenging problem [14]-[17]. Models of varying complexity have been used to approximate the dynamics of friction [14]. However, under practical conditions, the adequacy of the model for the control system to yield effective friction compensation has always remained questionable. Other efforts have been made toward more advanced control of precision motion systems. In [18], a neural-network (NN) based feedforward assisted proportional integral derivative (PID) controller was proposed. A hybrid control strategy using a variable structure control (VSC) is suggested for submicron positioning control [19]. These methods need an explicit system model for the control design, and the performance achievable depends on the accuracy of the model.

In this paper, we propose a learning-enhanced nonlinear PID control strategy for an indirect-drive ultrasonic-type of LPM. The control scheme does not require an accurate system model, but it is capable of yielding good motion tracking performance, as evident in the simulation study and experiments presented 


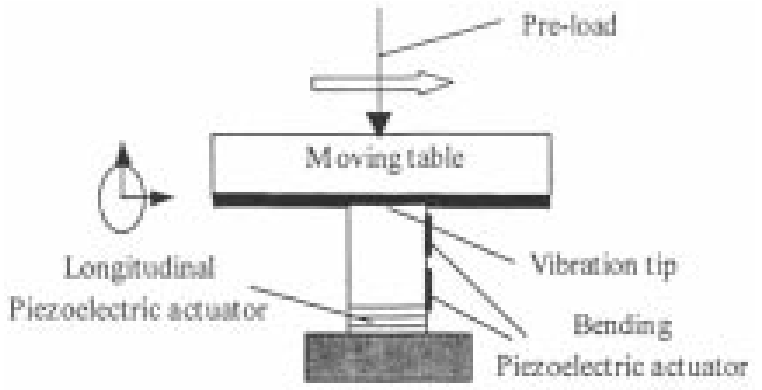

Fig. 1. Ultransonic-type LPM.

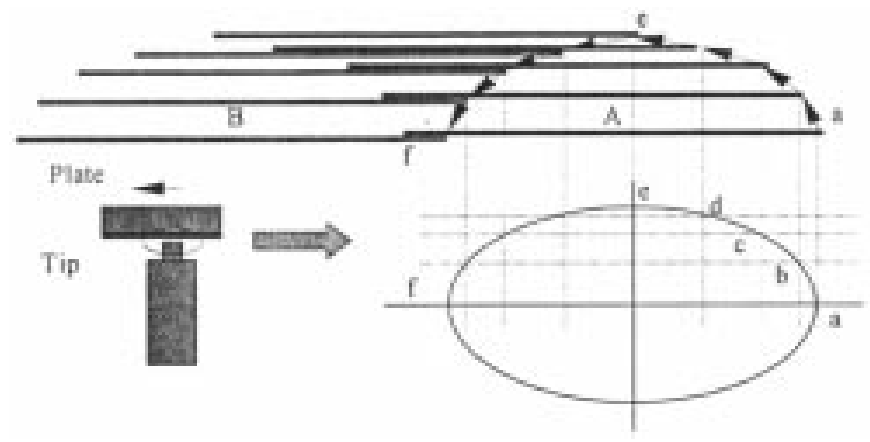

Fig. 2. Elliptical motion cycle.

in the paper. The nonlinear PID controller is composed of two tracking differentiators (TDs) which can yield high quality differential signal in the presence of disturbances and measurement noise. With an additional learning controller, the maximum position tracking error can be further reduced by approximately $50 \%$. As will be illustrated in the results forthcoming from the simulation study and experiments, the control system is robust to the presence of disturbances and measurement noises. Above all, the structure of the control system is simple and directly intuitive to the practitioners.

\section{MODELING OF LPM}

Fig. 1 shows the principal structure of an ultrasonic-type LPM considered in this work.

The stator vibrator is fitted with bending and longitudinal piezoelectric actuators. They are driven by two electrical sources of identical frequency, but with a phase difference that is carefully controlled. At the vibration tip, an elliptical motion is thus created, resultant of the elliptical and longitudinal motion. The bending actuators convert a large electrical power to mechanical output and the longitudinal actuator dynamically changes the force along the pre-load direction to adjust the frictional force between the stator and the rotor. A vibration circuit working at resonant frequency is used to cause the longitude and bending ceramic components to vibrate.

Fig. 2 shows the detailed operational process in a single elliptical motion cycle.

Assume the tip of the piezoelectric element, in contact with the moving plate, is initially at point $a$. It begins an elliptical motion from this point, moving to point $f$ via points $b, c, d$, and $e$ in order. This motion will result in a translation of the plate from

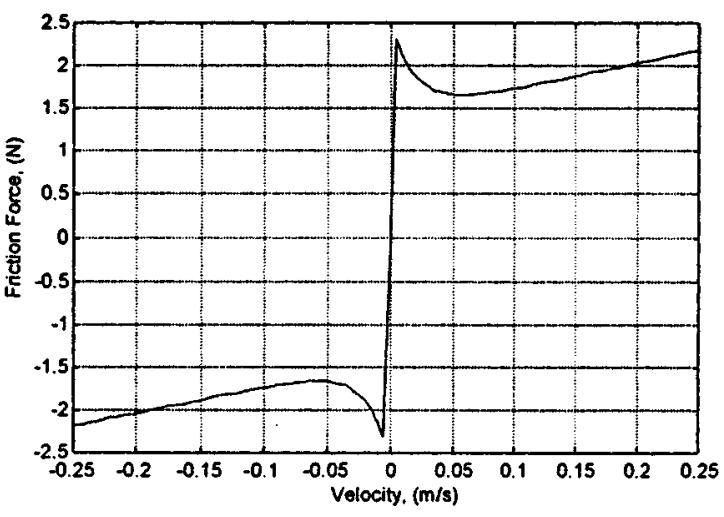

Fig. 3. Friction model.

$A$ to $B$. Subsequently, the tip of the piezoelectric element returns to point $a$ from $f$, but there is no further resultant plate motion since there is no contact made for this part of the motion. This completes one motion cycle, and subsequent cycles are similarly iterated. The velocity and direction of the moving plate can be adjusted by changing the shape and phase of the ellipse. Thus, there is a complicated function of frictional force acting during a complete motion cycle.

Friction is inevitable in many practical systems and its effect on machine performance has been demonstrated by a number of researchers [14]. It is mostly noticeable at low velocity because of the extremely high negative gain at velocities near zero. It is a highly complicated process to attempt to build an explicit mathematical friction model for the LPM because friction plays a dual role: it does not simply contribute to the nonlinear dynamics (e.g., dead zone) of the LPM, but it also serves as the driving force for the moving part. For the purpose of simulation, a conventional and generally acceptable Tustin friction model [14] will be adopted. It should be noted that the model is simulated as part of the dynamics of the LPM. The control system does not require the friction model.

The LPM can be expressed as a second-order nonlinear differential equation

$$
m \ddot{y}=u-K_{\mathrm{vf}} \dot{y}-f(\dot{y})
$$

where $m$ denotes the total moving mass, $y$ denotes the position, $u$ denotes the applied force, and $K_{\mathrm{vf}}$ denotes the viscous damping coefficient. $K_{\mathrm{vf}} \dot{y}$, thus, represents the viscous friction and $f(\dot{y})$ represents the combined effects of negative viscous and the Coulomb friction. The Tustin model for $f(\dot{y})$ is given by

$$
f(\dot{y})=\left(a_{0}+a_{1} e^{-a_{2}|\dot{y}|}\right) \operatorname{sgn}(\dot{y})
$$

where $a_{0}, a_{1}$, and $a_{2}$ are the three parameters of the model and $\operatorname{sgn}($.$) represents the standard sign operator.$

Fig. 3 shows the friction characteristics around the static point. It can be seen that there is a large force variation for small variation of the velocity near zero.

Fig. 4 shows the SIMULINK block diagram of the LPM. First order estimates of $K_{\mathrm{f}}$ (force constant) and $K_{\mathrm{vf}}$ (velocity 


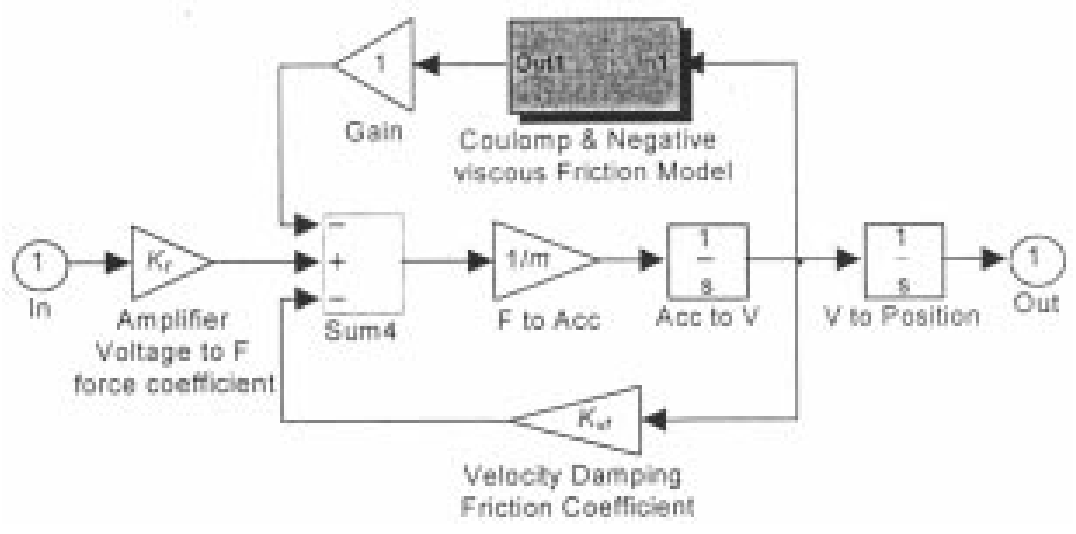

Fig. 4. SIMULINK block diagram.

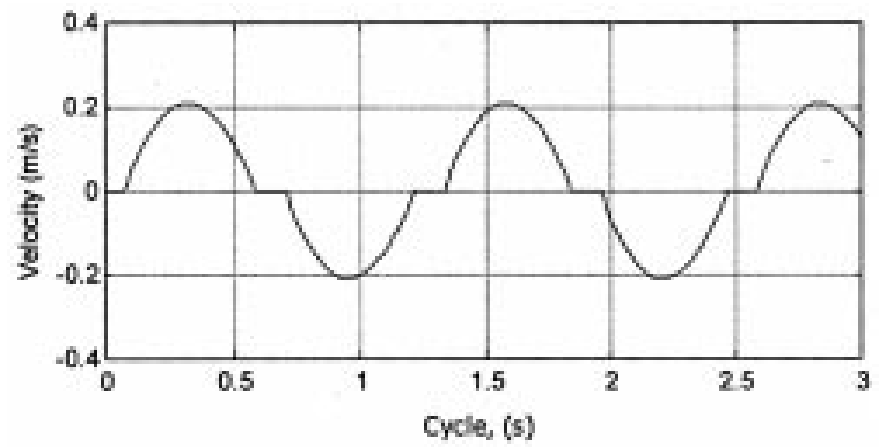

Fig. 5. Velocity response.

damping coefficient) can be obtained from simple step experiments as

$$
\left\{\begin{array}{l}
K_{\mathrm{vf}}=\frac{m}{\tau} \\
K_{\mathrm{f}}=\left|V_{\infty}\right| \frac{K_{\mathrm{vf}}}{U_{\mathrm{in}}}
\end{array}\right.
$$

where $U_{\text {in }}$ is the step size of a step input signal; $V_{\infty}$ is the corresponding steady-state velocity of the step response, and $\tau$ is the system time constant obtained from the same step response.

The simulated velocity response to a sine input signal for the nonlinear system is shown in Fig. 5.

It should be reiterated that the model presented in this section is used mainly in the simulation of the actual LPM, when assessing the performance of the control system. The control system does not need an accurate or full model in its design. The next section will elaborate on the details.

\section{Controller Design}

The conventional PID controller is a widely used industrial controller which uses a combination of proportional, integral, and derivative action on the control error to form the output of the controller. It is known that a linear combination of these components can achieve a compromised performance in terms of system response speed and stability. A nonlinear combination can provide additional degree of freedom to achieve a much improved system performance [20]. However, this improvement is achieved at the expense of higher complexity in the controller.
Artificial intelligence approaches can alleviate some of the difficulties by fusing a priori information or expert knowledge into the control design.

Another prominent difficulty with PID control is in the practical implementation of the derivative action. Derivative action provides a degree of predictive control capability to yield faster response without an excessive overshoot/undershoot, its practical merits are often questionable. In the presence of measurement noise or rapidly changing disturbance signals, it is often unclear as to whether derivative action will give any control improvement. In some cases, a first-order filter is used in conjunction with the differentiator. In other cases, derivative action may just be switched off altogether. In this section, we adopt a nonlinear TD to replace the differential component of the PID controller for more effective and robust performance in the presence of noise and other uncertainties. The TD can be further expanded to serve as an extension states observer (ESO). The ESO can act as a soft sensor of general disturbance signals arising in the control system. A feedforward control action may then be taken in response to the observed disturbance before it affects the system performance. The concepts behind the ESO and TD have been proposed in a general empirical form [20]-[22]. While a rigorous analysis of their stabilities has not been available, the viability for practical applications has been demonstrated via simulation and experiments [20]-[22]. Feng [23], [24] has specifically applied these concepts to speed control of induction motor.

In this paper, we will embed these concepts in the formulation of a nonlinear PID controller, and demonstrate its performance on a LPM for precise positioning control applications. The nonlinear PID control system will be augmented with a repetitive learning control scheme for further performance enhancement when the system executes repetitive operations. The error states from previous iterations are used in a PID-type learning law to produce an additional feedforward control output. As we will show in the simulation study and experiments, the learning feed forward control component can achieve arbitrarily good performance. It only requires the feedback control system to be stable, although a well-tuned feedback controller will help to expedite the learning convergence.

Details of the various control components will be elaborated on in the pursuing subsections. 


\section{A. Nonlinear Linear Tracking Differentiator}

Consider a system described by

$$
\left\{\begin{array}{l}
\dot{z}_{1}=z_{2} \\
\dot{z}_{2}=f\left(z_{1}, z_{2}\right)
\end{array}\right.
$$

has solutions meeting the requirements: $z_{1}(t) \rightarrow 0, z_{2}(t) \rightarrow 0$, when $t \rightarrow \infty$. Then, for any boundary integrable functions $v(t)$, the solution, $x_{1}(t)$, of the system

$$
\left\{\begin{array}{l}
\dot{x}_{1}=x_{2} \\
\dot{x}_{2}=R^{2} f\left(x_{1}-v, x_{2} / R\right)
\end{array}\right.
$$

will satisfy

$$
\lim _{R \rightarrow \infty} \int_{0}^{T}\left|x_{1}(t)-v(t)\right| d t=0
$$

where $R$ is a constant.

One form of TDs, which meet above requirements, is as follows:

$$
\left\{\begin{array}{l}
\dot{x}_{1}=x_{2} \\
\dot{x}_{2}=-R \operatorname{sgn}\left(x_{1}-v(t)+\frac{\left|x_{2}\right| x_{2}}{2 R}\right) .
\end{array}\right.
$$

To reduce the phenomenon of oscillation, the function $\operatorname{sgn}()$ may be replaced by function sat(), where

$$
\operatorname{sat}(A, \delta)= \begin{cases}\operatorname{sgn}(A), & |A|>\delta \\ \frac{A}{\delta}, & |A| \leq \delta, \quad \delta>0\end{cases}
$$

and $\delta$ is a small constant and $A=x_{1}-v(t)+\left|x_{2}\right| x_{2} / 2 R$.

The system described by (7) becomes

$$
\left\{\begin{array}{l}
\dot{x}_{1}=x_{2} \\
\dot{x}_{2}=-R \operatorname{sat}(A, \delta) .
\end{array}\right.
$$

The system described by (8) can be used as a high performance TD of the tracking signal $v(t)$, and the differential component in conventional PID controller can be replaced by the TD for a more robust performance. Fig. 6 shows the performance of the TD, when $v(t)=20 \sin (\omega \cdot t)$ and it is perturbed by an additive white noise component with the maximum amplitude of 0.5. For clear illustration, the differential signals obtained, respectively, by the TD and the general PID controller are normalized by $\omega$ in Fig. 6. It can be seen that the differential tracking performance of TD is much better compared to that of a pure differentiator.

The design parameters can be selected empirically. In the simulation and experiments, the empirical rule, $\delta=0.00005 R$ works well. Improved differential tracking performance can be achieved by increasing the value of $R$. However, an increase in $R$ also leads to more significant high frequency noise. Thus, the choice of $\mathrm{R}$ is a tradeoff between tracking performance and noise rejection, and should be selected carefully with respect to the actual requirements.

\section{B. Nonlinear ESO}

Suppose the system model is described by

$$
y^{(n)}=f(\cdot)+d(t)+k u(t)
$$

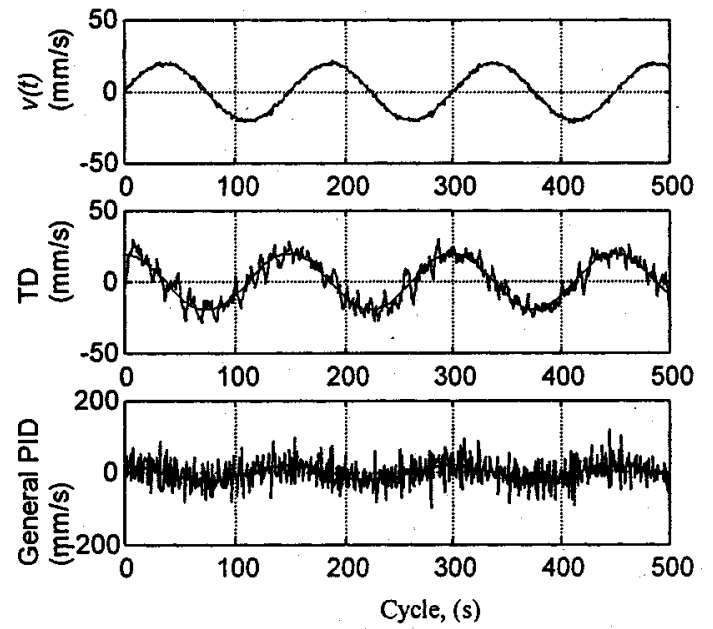

Fig. 6. Differential signals.

where $f(\cdot)=f\left(y, y^{(1)}, \ldots, y^{(n-1)}, t\right), d(t)$ is the disturbance signal, and $\mathrm{k}$ is a control coefficient. $u(t)$ and $y(t)$ denote the input and output of the system, respectively. A nonlinear state observer is designed to estimate system states, as well as an extension state [22]. The nonlinear observer for the system is described as

$$
\left\{\begin{array}{l}
\dot{z}_{1}=z_{2}-g_{1}\left(z_{1}-y\right) \\
\dot{z}_{2}=z_{3}-g_{2}\left(z_{1}-y\right) \\
\quad \vdots \\
\dot{z}_{n-1}=z_{n}-g_{n-1}\left(z_{1}-y\right) \\
\dot{z}_{n}=z_{n+1}-g_{n}\left(z_{1}-y\right)+k u(t) \\
\dot{z}_{n+1}=-g_{n+1}\left(z_{1}-y\right)
\end{array}\right.
$$

where, $z_{1}, z_{2}, \ldots, z_{n}$ are the estimated values of $y, \dot{y}, \ldots$, $y^{(n-1)}$, respectively, and $g_{1} \sim g_{n+1}$ are nonlinear compensation functions. The state, $z_{n+1}$, is called the extension state. Let

$$
\left[\begin{array}{c}
x_{1} \\
x_{2} \\
\vdots \\
x_{n}
\end{array}\right]=\left[\begin{array}{c}
y \\
\dot{y} \\
\vdots \\
y^{(n-1)}
\end{array}\right]
$$

Equation (9) may be rewritten in the following form

$$
\left\{\begin{array}{l}
\dot{x}_{1}=x_{2} \\
\dot{x}_{2}=x_{3} \\
\vdots \\
\dot{x}_{n}=a(t)+k u(t) \\
\dot{x}_{n+1}=b(t) \\
y(t)=x_{1}(t)
\end{array}\right.
$$

where $a(t)=f(\cdot)+d(t)$ and $b(t)$ is an unknown function.

Denoting $\delta_{1}=z_{1}-x_{1}, \ldots, \delta_{n+1}=z_{n+1}-x_{n+1}$, it follows that

$$
\left\{\begin{array}{l}
\dot{\delta}_{1}=\delta_{2}+g_{1}\left(z_{1}-x_{1}\right) \\
\dot{\delta}_{2}=\delta_{3}+g_{2}\left(z_{1}-x_{1}\right) \\
\quad \vdots \\
\dot{\delta}_{n-1}=\delta_{n}+g_{n-1}\left(z_{1}-x_{1}\right) \\
\dot{\delta}_{n}=a(t)-z_{n+1}+g_{n}\left(z_{1}-x_{1}\right) \\
\dot{\delta}_{n+1}=b(t)+g_{n+1}\left(z_{1}-x_{1}\right) .
\end{array}\right.
$$




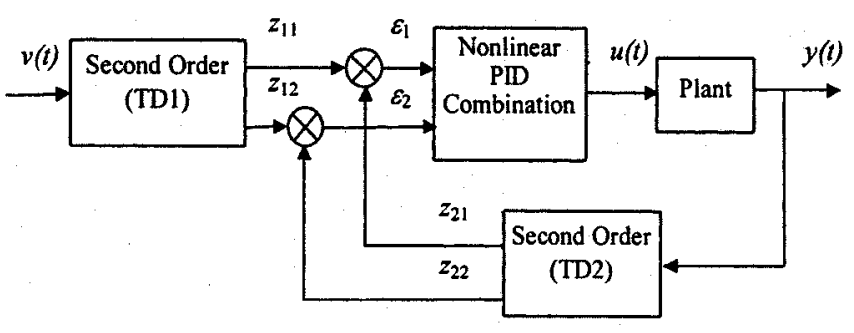

Fig. 7. Nonlinear PID controller.

It has been shown in [19] the functions $g_{i}(), i=1, \ldots, n+1$ can be chosen to stabilize the system (13) at the operating point. Then, if the system is stable, for suitable nonlinear functions, $g_{1} \sim g_{n+1}$, it follows that

$$
\lim _{t \rightarrow \infty}\left|a(t)-z_{n+1}\right|=\varepsilon
$$

where $\varepsilon$ is an arbitrarily small positive constant. Equation (14) means that the extension state, $z_{n+1}$, is the estimated value of $f(t)+d(t)$, which is really the uncertain value of the system to be estimated. The nonlinear observer's structure (10) is not determined by the actual expression of $y^{(n)}(t)$, but only affected by the variation range of $b(t)=y^{(n+1)}(t)$. The fal $($.$) function$ (see Section III-C) will be used as the nonlinear compensation functions, $g_{i}, i=1,2, \ldots, n+1$.

\section{Nonlinear PID Control}

With two second-order TDs, a nonlinear PID controller can be constructed as in Fig. 7.

TD1 and TD2 are two TDs which track input signal $v(t)$, output signal $y(t)$ and their differentials. Variables, $\mathrm{z}_{11}, \mathrm{z}_{21}, \mathrm{z}_{12}$, and $z_{22}$ are their estimated values. $\varepsilon_{1}$ and $\varepsilon_{2}$ denote the position and velocity tracking errors, respectively. Analogous to general linear PID controller, the nonlinear combination of the error signals can be expressed as

$$
u(t)=k_{p} \operatorname{fal}\left(\varepsilon_{1}, \alpha, \delta\right)+k_{i} \int \varepsilon_{1} d t+k_{d} \operatorname{fal}\left(\varepsilon_{2}, \alpha, \delta\right)
$$

where $k_{p}, k_{i}$, and $k_{d}$ are the PID control coefficients, fal(.) is a selected nonlinear function described by

$$
\operatorname{fal}(\varepsilon, \alpha, \delta)= \begin{cases}|\varepsilon|^{\alpha} \operatorname{sgn}(\varepsilon), & |\varepsilon|>\delta \\ \frac{\varepsilon}{\delta^{1-\alpha}}, & |\varepsilon| \leq \delta\end{cases}
$$

where $\alpha, \delta$ are constants.

Fig. 8 shows the characteristics of the fal () function. A linear relationship is thus effectively used when $\varepsilon \leq|\delta|$ to provide a smoother control action when $\varepsilon$ is near zero.

Empirically, the design parameters may be selected according to the guidelines $\delta=0.1 \sim 0.2, R>100000, \alpha=0.5 \sim 1, k_{p}$, $k_{i}$, and $k_{d}$ can be adjusted accordingly, following the many rules available for standard PID control design.

Using a third-order ESO, an improved version of the nonlinear PID controller (Fig. 9) can be constructed which can deal more effectively with disturbance signals $\mathrm{d}(\mathrm{t})$ arising in the system. This system is called auto-disturbance rejection controller (ADRC) [23].

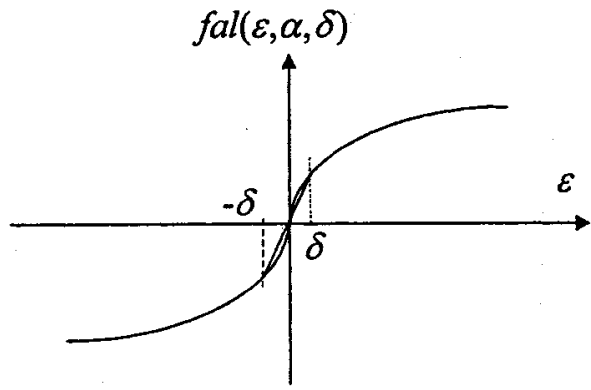

Fig. 8. fal () function.

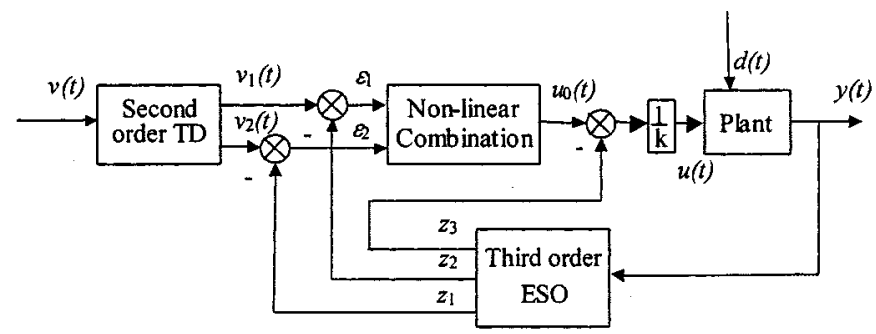

Fig. 9. Nonlinear state feedback controller.

The equation for nonlinear combination can be expressed as

$u_{0}(t)=\beta_{1} \operatorname{fal}\left(\varepsilon_{1}, \alpha, \delta\right)+k_{i} \int \varepsilon_{1} d t+\beta_{2} \operatorname{fal}\left(\varepsilon_{2}, \alpha, \delta\right)$.

The control law is thus

$$
u(t)=\frac{u_{0}(t)-z_{3}}{k}
$$

where $z_{3}$ is provided by the state estimator (10).

The nonlinear PID controller proposed has a more general form compared to the linear PID controller, with more degreeof-freedom in tuning the controller. However, how much performance improvement can be achieved also depends on the choice of the control parameters. The constant PID gains $k_{p}, k_{i}$, and $k_{d}$ can be selected using the many PID tuning rules available for linear PID control. Empirical rules are also suggested for the nonlinear part of the controller in this section. A learning approach will be further proposed in the next section to yield efficient control when the task to be executed is repetitive in nature.

\section{Repetitive Learning Control}

In many applications, the LPM can be subject to periodic reference input signal. For example, for a precision CNC elliptical piston-turning machine, a high speed, high precision linear actuator is needed to track a $40-\mathrm{Hz}$ periodic signal with a stroke of $0.5 \mathrm{~mm}$ [25]. For high performance engine pistons, a maximum contour error of $4 \mu \mathrm{m}$ is required. A similar application is found in [26].

The key idea of repetitive learning control is that the error signals of previous periods are used to reduce the current error caused by the periodic reference input. A precise mathematical model, which is difficult to obtain, is not absolutely required.

In applications involving repetitive operations, discrete time repetitive learning control technology for LPM to improve the 


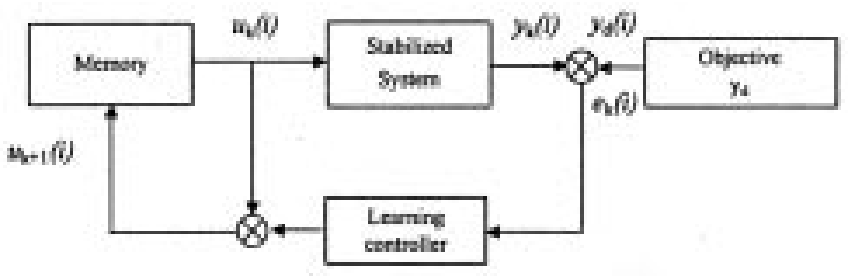

Fig. 10. Repetitive learning control.

TABLE I

SPECIFICATIONS OF LPM

\begin{tabular}{l|c|c|c|c}
\hline Stroke & $\begin{array}{c}\text { Velocity } \\
\text { (Max) }\end{array}$ & $\begin{array}{c}\text { Resoluti } \\
\text { on }\end{array}$ & Input & $\begin{array}{c}\text { Output } \\
\text { force } \\
\text { (Max) }\end{array}$ \\
\hline $170 \mathrm{~mm}$ & $250 \mathrm{~mm} / \mathrm{s}$ & $0.4 \mu \mathrm{m}$ & $+1-10 \mathrm{~V}$ & $40 \mathrm{~N}$ \\
\hline
\end{tabular}

response to periodic reference inputs will be tested. As shown in Fig. 10, the learning controller represents an additional feedforward control branch to the stabilized system which is controlled by the nonlinear PID controller described in the earlier subsections.

A PID-type learning law is used for learning controller. The learning law is described by

$$
u_{k+1}(i)=u_{k}(i)+f\left(e_{k}(i), \sum_{i} e_{k}(i), \frac{d e_{k}(i)}{d t}\right)
$$

where $u_{\mathrm{k}+1}(i)$ is the learning control signal generated during the $(k+1)$ th cycle for the $i$ th sampling point; $f($.$) is the cor-$ rection function for the learning value. A proposed PID-type correction function is given by

$$
\begin{array}{r}
f(\cdot)=k_{P} e_{k}(i+1)+k_{I} \sum_{n=1}^{i+1} e_{k}(n)+k_{D}\left[e_{k}(i+1)-e_{k}(i)\right], \\
i \in[0, N-1]
\end{array}
$$

where $N$ is the number of sample points within a cycle.

\section{SimUlation AND EXPERIMENT}

To illustrate the effectiveness and applicability of the control scheme developed, simulation and real-time experiments are carried out on a single axis linear stage manufactured by Steinmeyer. The stage is driven by a SP-8 piezoelectric motor which is manufactured by Nanomotion. Table I shows the specifications of the stage and the motor. The DSPACE DS1102 control card is used in conjunction with MATLAB and SIMULINK for the experiment.

The following system model is used for simulation:

$$
\left\{\begin{array}{l}
0.45 \ddot{y}=3.076 u-39.13 \dot{y}-6.142 \operatorname{sgn}(\dot{y})-f(\dot{y})-f_{d} \\
f(\dot{y})=4.9216 e^{-86.956|\dot{y}|} \operatorname{sgn}(\dot{y}) .
\end{array}\right.
$$

\section{A. Simulation Results}

A sinusoidal reference signal, $v(t)=1.5 \sin (30 \pi \cdot t)$, is used for the simulation. In addition, a disturbance signal described by

$$
d(t)=0.2 \times 1.5 \times \operatorname{sgn}(\sin (60 \pi \cdot t))
$$

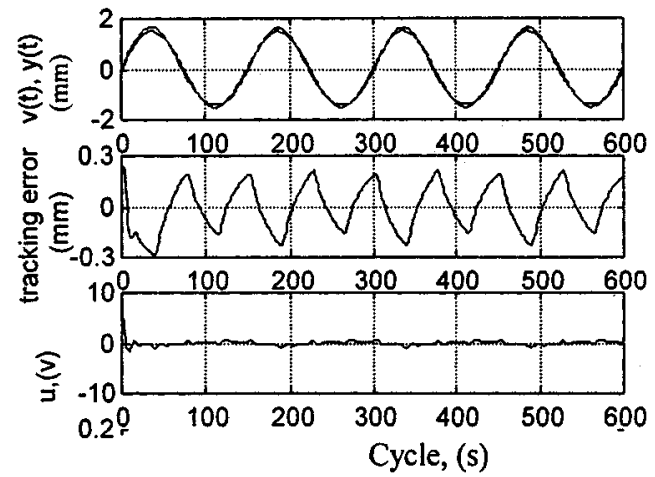

Fig. 11. Linear PID, under disturbance, $d(t)$.

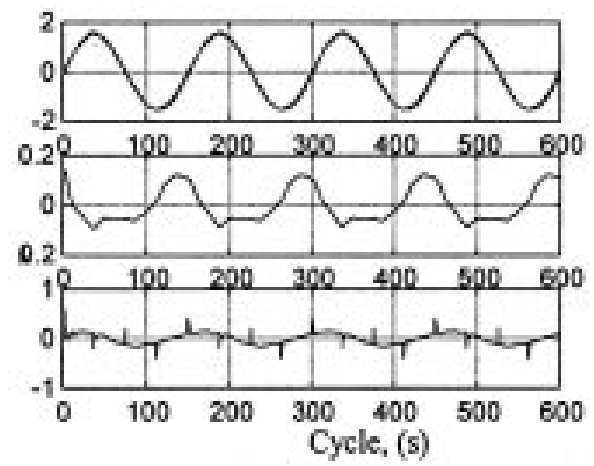

Fig. 12. Nonlinear PID with ESO under disturbance, $d(t)$.

is simulated. The simulation results based on the use of a linear PID controller and the nonlinear PID controller with the ESO are shown in Figs. 11 and 12, respectively. For a fair comparison, the linear PID controller is fine tuned to yield optimal performance.

The nonlinear controller with the ESO exhibits an improved tracking and disturbance rejection performance compared to the linear PID controller. The maximum tracking error is reduced by $50 \%$.

Finally, a learning controller is added to improve the tracking precision. Figs. 13 and 14 show the control results with the learning controller augmented. A maximum tracking error of \pm 0.002 can be achieved after 30 cycles in the absence of disturbances and \pm 0.01 in the presence of disturbances. The error curve is now completely different from the one of Fig. 12 due to the additional learning control component. Although it is still periodic, the frequency is changed.

Fig. 15 shows the error convergence curve. The error convergence speed is dependent on the PID parameters of the learning controller. In fact, the coefficients $k_{p}, k_{i}$, and $k_{d}$ can be changed from one cycle to another to improve the system convergence performance. Thus, for example, larger parameters for the learning controller may be applied to obtain high convergence speed initially, and they may be subsequently reduced to obtain higher tracking precision.

\section{B. Experimental Results}

The real-time experimental results obtained on the physical system are provided categorically below. 


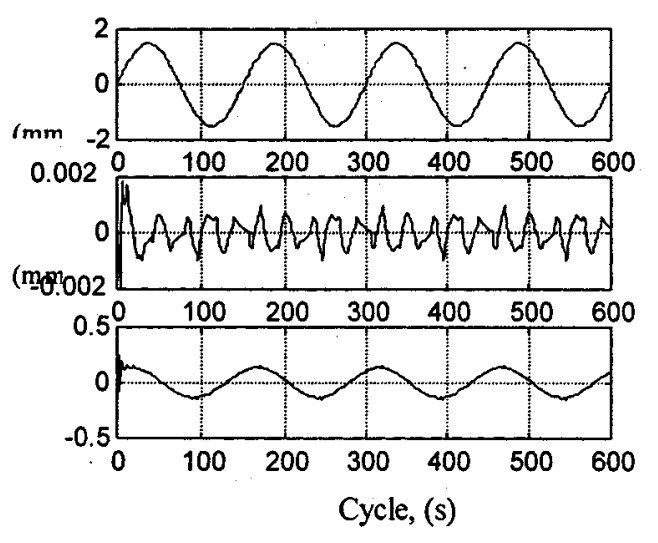

Fig. 13. Learning nonlinear controller.

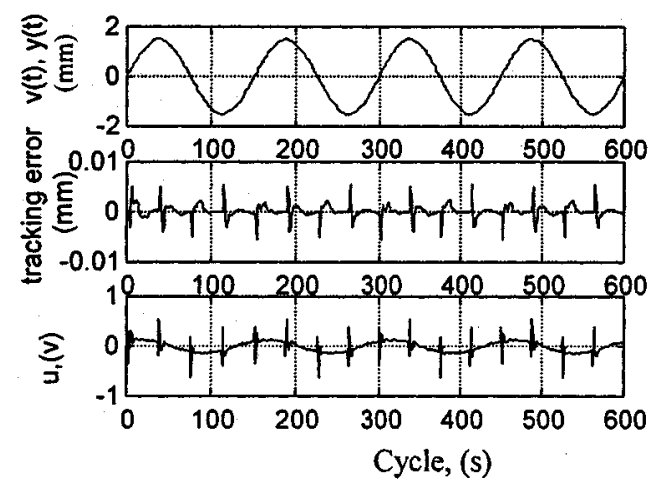

Fig. 14. Learning nonlinear controller under disturbance, $d(t)$.

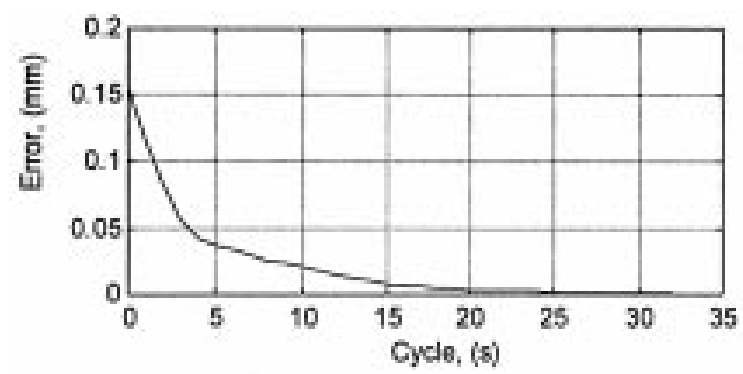

Fig. 15. Error convergence curve.

1) TD Performance: Fig. 16 shows the differential output signals obtained using the TD and a pure differentiator $(d y / d t)$ (with a low-pass filter) when a white noise disturbance $d(t)$ appears at the input. Although improvement is observed in the TD which gives a smoother differential signal, it is not very significant as the mechanical system acts as an inherent high frequency filter to the input signal. An interesting observation is that the experimental results in Fig. 16) are very similar to the simulation results (Fig. 5), thereby verifying to some extent the adequacy of the model adopted.

When the disturbance appears at the output, the improvement using TD is clearly evident from the respective signals in Figs. 17 and 18. A clean differential signal is obtained from the TD. From the pure differentiator $(d y / d t)$, the profile of the differential signal is almost completely lost in noise.

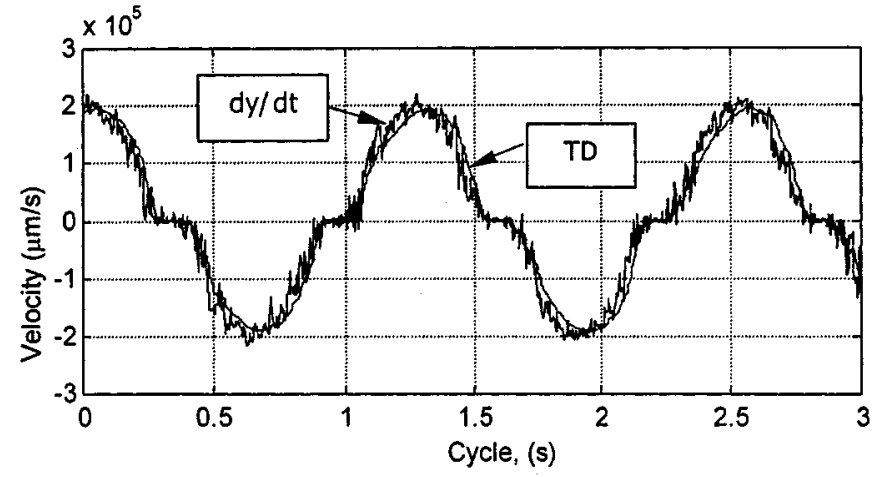

Fig. 16. Differential signals.

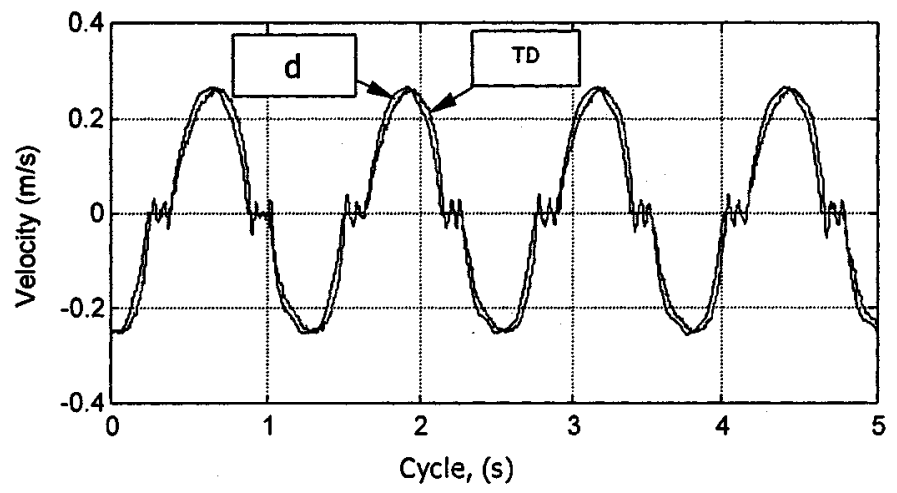

Fig. 17. Differential signals from DT. Curve $d$ is the ideal differential signal.

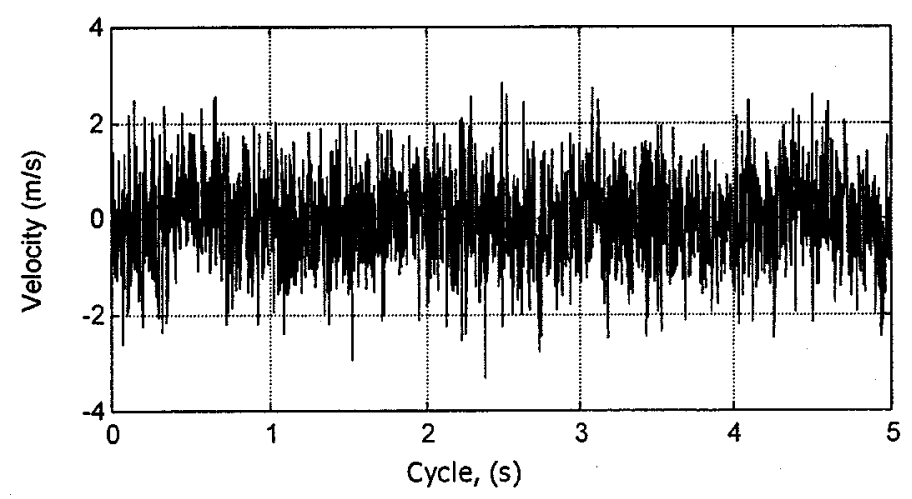

Fig. 18. Differential signal from $\mathrm{dy} / \mathrm{dt}$.

2) Nonlinear PID Controller Performance: Figs. 19 and 20 show the system tracking errors resultant of using the nonlinear PID controller and the linear PID controller. The tracking error from the nonlinear PID controller is about 50\% that from the linear PID controller. The maximum error occurs at the transition point when the motion changes direction and the full dosage of friction is experienced.

3) Learning Nonlinear PID Performance: Next, a learning mode is added to the nonlinear PID controller. Fig. 21 shows the results. The tracking error in Fig. 21 is obtained at the 20th learning cycle, which is a further $50 \%$ of the tracking error obtained from a nonlearning NPID controller. Fig. 22 shows the control output of the controller. The sign of the control output is changed when the motion switches direction. 


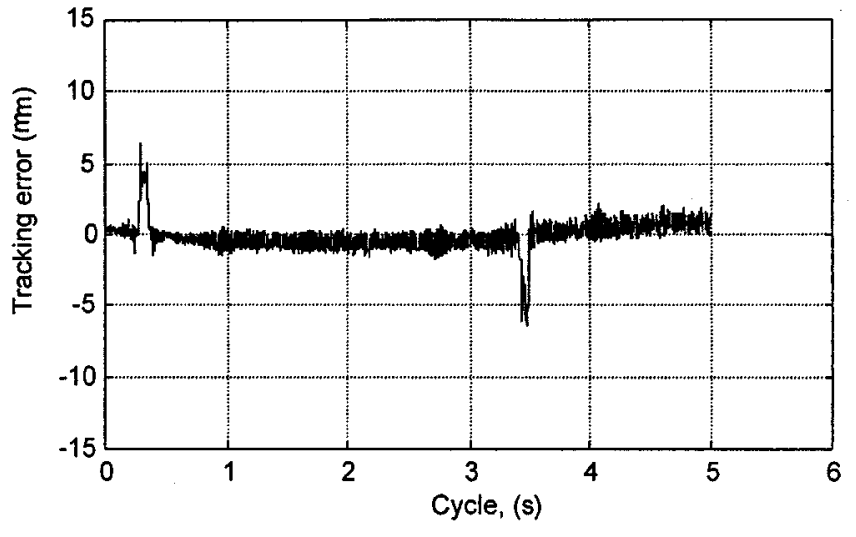

Fig. 19. Tracking error-Nonlinear PID control.

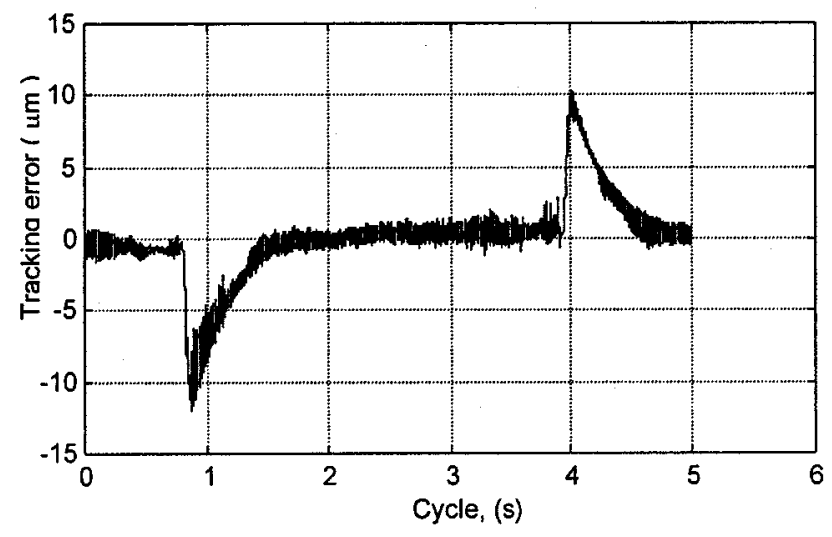

Fig. 20. Tracking error-Linear PID.

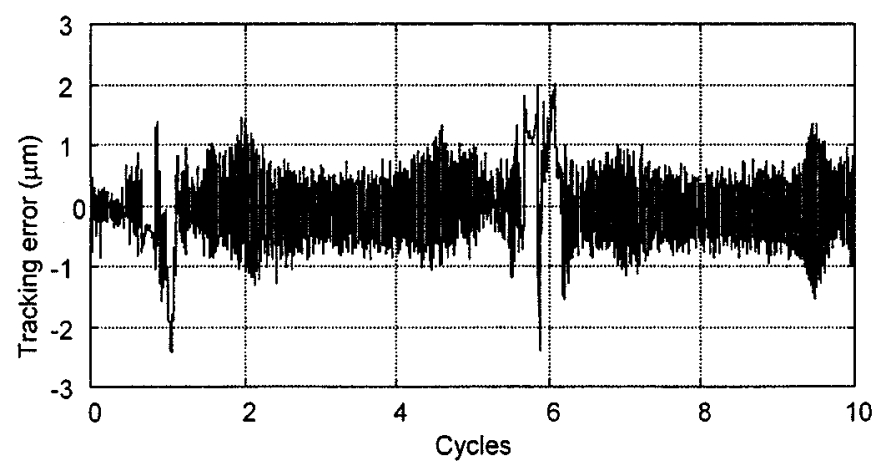

Fig. 21. Tracking error-20th cycle.

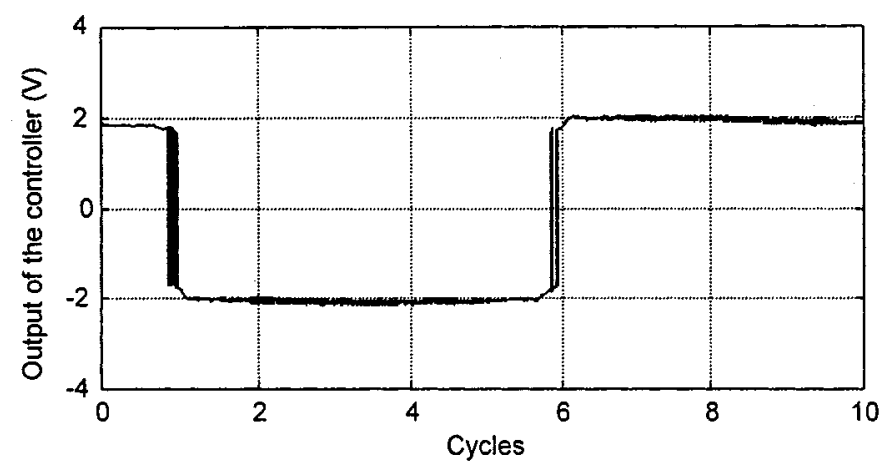

Fig. 22. Control output.

\section{CONCLUSION}

In this paper, we have demonstrated the effectiveness of a nonlinear PID controller augmented with a learning strategy when applied to precision motion control of piezoelectric actuators. The nonlinear PID controller is composed of two TDs which can yield high quality differential signal in the presence of disturbances and measurement noise. With an additional learning controller, the maximum position tracking error can be further reduced by approximately $50 \%$.

\section{REFERENCES}

[1] A. Visscher and J. W. Coenders, "Linear piezo motor," Philips Center for Manufacturing Technology, Eindhoven, The Netherlands, Internal Rep. CTR595-93-0147, 1993.

[2] T. Moriwaki and E. Shamoto, "Ultraprecision feed system based on walking drive," Annals CIRP, vol. 46, no. 1, pp. 505-508, Jan. 1997.

[3] J. Kim and D. Kim, "Development and application of an ultraprecision lathe," Int. J. Adv. Manufact. Technol., vol. 13, pp. 164-171, 1997.

[4] Z. Wang, M. K. Jouaneh, and D. A. Dornfeld, "Design and characterization of a linear motion piezoelectric micropositioner," IEEE Control Syst. Mag., pp. 10-15, Feb. 1990.

[5] B. Zhang and Z. Zhu, "Developing a linear piezomotor with nanometer resolution and high stiffness," IEEE/ASME Trans. Mechatron., vol. 2, pp. 22-29, Mar. 1997.

[6] M. J. Edward, "Piezoelectric/magnetostrictive resonant inchworm motor development," Ph.D. dissertation, George Washington Univ., Washington, DC, Nov. 1994.

[7] M. Takahashi, M. Kurosawa, and T. Higuchi, "Direct friction driven surface acoustic wave motor," in Proc. 8th Int. Conf. Solid-State Sensors and Actuators, Stockholm, Sweden, June 1995, pp. 401-404.

[8] S. Ueha and Y. Tomikawa, Ultrasonic Motors-Theory and Applications. Oxford, U.K.: Clarendon Press, 1993.

[9] M. Kurosawa and S. Ueha, "Hybrid transducer type ultrasonic motor," IEEE Trans. Ultrason., Ferroelect., Freq. Contr., vol. 38, pp. 89-92, Mar. 1991.

[10] D. Newton, E. Garcial, and G. C. Horner, "A linear piezoelectric motor," Smart Materials and Structures, no. 6, pp. 295-304, 1997.

[11] M. Kummel, S. Goldschmidt, and J. Wallaschek, "Theoretical and experimental studies of a piezoelectric ultrasonic linear motor with respect to damping and nonlinear material behavior," Ultrason., vol. 36, pp. 103-109, 1998.

[12] P. Ge and M. Jouaneh, "Tracking control of a piezoceramic actuator," IEEE Control Syst. Mag., vol. 4, pp. 209-216, May 1996.

[13] M. Krstic, I. Kanellakopoulos, and P. Kokotovic, Nonlinear and Adaptive Control Design. New York: Wiley, 1995.

[14] B. Armstrong-Helouvry, P. Dipont, and C. Canudas de Wit, "A survey of models, analysis tools and compensation methods for the control of machines with friction," Automat., vol. 30, pp. 1083-1138, 1994.

[15] H. D. Taghirad and P. R. Belanger, "Robust friction compensator for harmonic drive transmission," in Proc. 1998 IEEE Int. Conf. Control Applications, 1998, pp. 547-551.

[16] E. W. Bai, "Parameterization and adaptive compensation of friction forces," Int. J. Adapt. Control Signal Process., vol. 11, pp. 21-31, 1997.

[17] C. Candas De Wit and P. Lischinsky, "Adaptive friction compensation with partially known dynamic friction model," Int. J. Adapt. Control Signal Process., vol. 11, pp. 65-80, 1997.

[18] G. Otten, T. J. A. de Vries, J. van Amerongen, A. M. Rankers, and E. W. Gaal, "Linear motor motion control using a learning feedforward controller," IEEE/ASME Trans. Mechatron., vol. 2, pp. 179-187, Sept. 1997.

[19] S. B. Chang, S. H. Wu, and Y. C. Hu, "Submicrometer overshoot control of rapid and precise positioning," J. Amer. Soc. Precision Eng., vol. 20, pp. 161-170, 1997.

[20] J. Han, "A new type of controller: NLPID" (in Chinese), Control and Decision, vol. 9, no. 6, pp. 401-407, Nov. 1994.

[21] — "Extension state observer for a type of uncertain objectives" (in Chinese), Control and Decision, vol. 10, no. 1, pp. 85-88, Feb. 1995.

[22] Z.-G. Hou, Y. Huang, and J. Han, "Active noise cancellation with a nonlinear control mechanism," in Proc. 37th IEEE CDC, Tampa, FL, 1998.

[23] G. Feng, L. Huang, and D. Zhu, "A nonlinear auto-disturbance rejection controller for induction motor," in Proc. IECON'98, vol. 3, 1998, pp. $1509-1514$.

[24] — - "A robust controller for improving disturbance rejection in speed control of induction motor," in Proc. IEEE PEDS'99, Hong Kong, 1999, pp. $432-437$. 
[25] H. Zhou, L. Zhang, and X. Wang, "Repetitive control and its application to linear servo unit for CNC machining of elliptical pistons," in Proc. IEEE ICIT'96, Shanghai, China, 1996, pp. 630-633.

[26] D. Kim and S. Kim, "An iterative learning control method with application for CNC machine tools," IEEE Trans. Ind. Applicat., vol. 32, pp. 66-72, Jan./Feb. 1996.

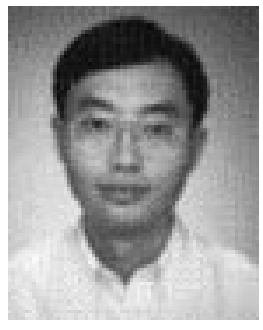

K. K. Tan (S'94-M'99) received the B.Eng. in electrical engineering (with honors) and the Ph.D. degree both from the National University of Singapore, Singapore, in 1992 and 1995, respectively.

His research interests include precision motion, intelligent control, and mechatronics.

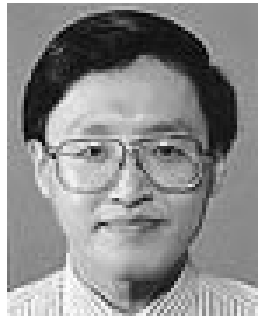

Tong Heng Lee (M'88) received the B.A. degree (first class honors) in the engineering tripos from Cambridge University, Cambridge, U.K., and the Ph.D. degree from Yale University, New Haven, CT, in 1980 and 1987, respectively.

$\mathrm{He}$ is a Professor in the Department of Electrical and Computer Engineering, National University of Singapore, Singapore. He is also the Head of the Control Engineering Division in the same department, and the Vice-Dean of Research in the Faculty of Engineering, National University of Singapore, Singapore. His research interests are in the area of adaptive systems, knowledge-based control, and intelligent mechatronics. He has published extensively in these areas and is currently an Associate Editor of Automatica, Control Engineering Practice, International Journal of Systems Science, and IEEE/ASME TRANSACTIONS ON MECHATRONICS and the IEEE TRANSACTIONS ON SYSTEMS, MAN, AND CYBERNETICS.

Prof. Lee was a recipient of the Cambridge University Charles Baker Prize in Engineering.

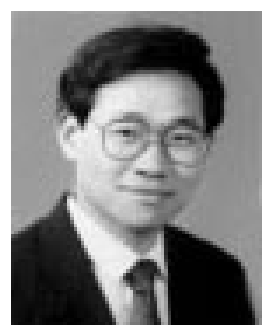

Huixing X. Zhou was born in Jiangsu, China, in 1963. He received the B.Eng. degree in production engineering from Dalian University of Technology, Dalian, China, and the Ph.D. degree in mechanical engineering from the Tsinghua University, Beijing, China, in 1983 and 1998, respectively.

From 1998 to 2000, he was a Postdoctoral Research Fellow in the Department of Electrical and Computer Engineering, National University of Singapore, Singapore. Since 2001, he has been a Product Development Manager in the Keyworth Institute, University of Leeds, Leeds, U.K. His research interests include CNC machine and DSP embedded system, motion control, linear actuators (piezo and VCM), mechatronics, and manufacturing systems. He is also interested in product development, technology transfer and management. 